
\title{
25 Research Soure \\ A Clinical Decision Support System for Prediction of Postpartum Hemorrhage in Vaginal Birth
}

\author{
Jingxuan Yang \\ Zhengzhou University \\ Peng Guo \\ Henan University of Technology \\ Yingli Song \\ Zhengzhou University \\ Lingli Han \\ Zhengzhou University \\ Xiaoyu Yang \\ Zhengzhou University \\ Hua Bai ( $\nabla$ sfyhlb3726@zzu.edu.cn ) \\ Zhengzhou University
}

\section{Research Article}

Keywords: postpartum hemorrhage, artificial neural network, clinical decision support system, prediction model, vaginal delivery

Posted Date: June 30th, 2021

DOI: https://doi.org/10.21203/rs.3.rs-618703/v1

License: (c) (i) This work is licensed under a Creative Commons Attribution 4.0 International License.

Read Full License 


\section{Abstract \\ Objective}

The morbidity and mortality caused by postpartum hemorrhage has been increased since 2016 in China, in addition, promoting vaginal delivery is an important task in China currently. This study aimed to develop a clinical decision support system (CDSS) to predict postpartum hemorrhage among vaginal delivery women.

\section{Design:}

A retrospective cohort study.

\section{Methods}

We performed a retrospective analysis of medical records among 1587 vaginal delivery women, who had visited the obstetrics clinic at the Third Affiliated Hospital of Zhengzhou University from 2018 to 2020, these women then were randomly divided into a training set (70\%), a validation set (15\%) and a test set (15\%). We adopted a univariate logistic regression model to select the significant features $(P<0.01)$. Afterward, we trained several artificial neural networks and binary logistic regression to predict the postpartum hemorrhage, the neural networks included multi-layer perceptron (MLP), back propagation (BP) and radial basis function (RBF). In order to compare and identify the most accurate network, we used the confusion matrix and the receiver operating characteristic (ROC) curve. We finally developed a clinical decision support system based on the most accurate network. All statistical analyses were performed by IBM SPSS (version 20), and MATLAB 2013b software was applied to develop the clinical decision support system.

\section{Results}

Initially, 45 potential variables were addressed by the univariate logistic regression, 16 significant predictors were then selected to enter the binary logistic regression and neural networks (P-value $<0.01)$. After validation, the best performing model was the multi-layer perceptron network with the highest discriminative ability (AUC $0.862,95 \% \mathrm{Cl} 0.838-0.887$ ). Followed by the back propagation model (AUC $0.866 ; 95 \% \mathrm{Cl} 0.842-0.890$ ), the logistic regression model (AUC 0.856; 95\% $\mathrm{Cl} 0.832-0.880$ ). The radial basis function model (AUC $0.845 ; 95 \% \mathrm{Cl} 0.820-0.870$ ) had lower discriminative ability.

\section{Conclusion}


In summary, in terms of predicting postpartum hemorrhage, the multi-layer perceptron network performed better than the back propagation network, logistic regression model, and radial basis function network. The developed clinical decision support system based on the multi-layer perceptron network is expected to promote early identification of postpartum hemorrhage in vaginal delivery women, thereby improve the quality of obstetric care and the maternal outcome.

\section{What Is Already Known About The Topic?}

- Postpartum hemorrhage is the leading cause of maternal mortality and morbidity worldwide.

- Due to the heterogeneity of discriminant criteria and risk factors between cesarean section and vaginal delivery, there are some limitations in predicting postpartum hemorrhage using the same warning model for different mode of delivery.

- Current prediction models for postpartum hemorrhage in vaginal delivery are linear algorithm that divide events into possible or not possible. However, most obstetrics events are not as black and white.

\section{What this paper adds}

- We developed the first midwifery-led clinical decision support system model based on artificial neural network (ANN) algorithm for prediction of postpartum hemorrhage in vaginal delivery.

- This study demonstrated that the multi-layer perceptron (MLP) network had the most accurate prediction ability, followed by the back propagation (BP) network, the logistic regression model, and the radial basis function (RBF) network.

- This study could enrich the practice of artificial intelligence in nursing informatization.

\section{Introduction}

Postpartum hemorrhage (PPH) is the leading cause of maternal mortality and morbidity worldwide, accounting for $29.3 \%$ of maternal deaths and $26.7 \%$ of adverse pregnancy outcomes, respectively ${ }^{[1-2]}$, it makes up $9.3 \%$ of maternal deaths in developed countries, while $45.7 \%$ in developing countries ${ }^{[3]}$. In developed countries, postpartum hemorrhage has fallen to the third as a direct cause of maternal death, after pregnancy hypertension and thromboembolism, however, it remains the leading cause of maternal death in developing countries ${ }^{[2]}$.

In China, with the opening of "two-child policy" from 2016, more and more elderly pregnant women have become the potential population of postpartum hemorrhage. The increasing trial of labor after a previous cesarean section (TOLAC) and elective repeated cesarean section (ERCS) caused by previous one-child policy, also deteriorate the risk of obstetric hemorrhage. Maternal mortality has been decreasing and seems to reach a bottleneck since 1998 in China, but the mortality caused by postpartum hemorrhage is 
increasing, compared with 2015 , the maternal mortality caused by postpartum hemorrhage increased by $15 \%$ in 2016 , to $23.5 \%$, and increased to $29.0 \%$ in $2017^{[1]}$.

The cesarean section rate in China rose from $34.1 \%$ in 2016 to $36.6 \%$ in $2019^{[1]}$, far from the $15 \%$ recommended by the World Health Organization ${ }^{[4]}$, thereby, promoting vaginal delivery is an important task in Chinese obstetrics department now. As a decision-maker for early warning of postpartum hemorrhage during the vaginal delivery, midwives often rely on their own experience to judge and have not yet formed a unified decision standard ${ }^{[5]}$. On the one hand, prediction and decision-making for postpartum hemorrhage in vaginal delivery is a formidable task for midwives. Multiple factors have varying influence on the occurrence of hemorrhage among vaginal delivery women, nonlinear relations between risk factors and postpartum hemorrhage make it more complicate as well ${ }^{[6]}$. On the other hand, the qualification of most Chinese midwives is college or technical secondary school education, and standardized training of midwives is low in China, as well.

The objective of this study is to develop a clinical decision support system (CDSS) to solve above problems. A clinical decision support system is invented to help physicians make appropriate decisions for patients, it uses artificial intelligence algorithm to solve semi-structured or unstructured clinical problem. Clinical decision support system not only is a principle and method based on the knowledge reasoning and logic operation to acquire and process relevant patient data from the electronic medical records automatically, but also is an auxiliary decision-making system which provide decision-makers with valuable information at the right time by the human-computer interaction ${ }^{[7]}$. Clinical decision support system is considered to be the inevitable trend of the combination of medicine and artificial intelligence ${ }^{[8-9]}$. Chinese government issued many policies to promote the sustainable development of medical informatization ${ }^{[10]}$, the notice of national hospital informatization construction focusing on electronic medical records by the National Health Commission in $2018^{[11]}$, pointed out that clinical nursing decision support system should realize intelligent input, intelligent generation, intelligent reminder, quality control and rectification of nursing records and other functions.

Data-driven clinical decision support system based on a large amount of electronic medical record data can be divided into case reasoning system and machine learning system, a good machine learning algorithms can extract personalized features from data and summarize patterns to achieve high accuracy ${ }^{[12]}$. Current algorithms of prediction model for postpartum hemorrhage adopted by the American College of Obstetricians and Gynecologists (ACOG) and the California Maternal Quality Care Collaborative (CMQCC) include logistic regression ${ }^{[13]}$, decision tree ${ }^{[14]}$, random forest and extreme gradient boosting ${ }^{[15]}$. Most of them are linear prediction that divides events into possible or not possible. However, as real life has shown, most events are not as black and white. Rather than other liner algorithms, artificial neural network (ANN) is a multi-layer complex model formed by connecting neurons (perceptron) through synapses (weights) ${ }^{[16]}$. ANN has strong nonlinear mapping ability to address highdimensional data, it can also reasonably predict and modify the relationship between input variables and output variables. ANN models possess strong feature of self-adaption, self-organizing, self-learning and 
tolerate faults ${ }^{[17]}$. In recent years, ANN has been applied to medical research, such as prediction of disease diagnosis and prognosis, prediction of nursing adverse events, which has proven high superiority [18-21].

Therefore, this study aims to build a scientific midwifery-led clinical decision support system based on ANN algorithm for prediction of postpartum hemorrhage in vaginal delivery, which will provide a practical theoretical basis for improving the success rate of treatment and reducing the mortality of postpartum hemorrhage.

\section{Material And Methods}

\subsection{Data source and data collection}

In order to identify the potential variables, we first conducted a literature system review and a Delphi method with obstetricians and midwives. We adopted the search strategy to find relevant literature as follows: (postpartum hemorrhage OR PPH OR transfusion) AND (predictor* OR risk factor*) AND (vaginal delivery OR vaginal birth) in PubMed, Web of Science, Embase, Springer, Elsevier SD and Wiley. We read related papers published in English from 2006. Moreover, we considered current medical evidence and obstetricians' consultations. We identified 54 potential variables regarding this; however, five of these 54 variables (residence, history of postpartum hemorrhage, pre-pregnancy weight, antenatal steroids, and antepartum infection) were not registered in the electronic medical records. Hence, we designed the data collection form and collected another 49 variables manually (Appendix 1). The needed data was retrospectively collected from the medical records of vaginal delivery women who had visited the obstetrics clinic at our hospital (the Third Affiliated Hospital of Zhengzhou University) in Zhengzhou, China from 2018 to 2020. We also tried to use the Maternal and Child Health Information Network of Henan Province to collect large data, but some essential elements were not available in the information system, so we collected complete data manually. Two trained researchers (first author and second author) extracted the data from the electronic medical records.

Out of 28765 registered vaginal deliveries from the electronic medical records, we excluded any patients who underwent (1) antenatal fetal demise, (2) gestations under 35 weeks, (3) caesarean section, (4) late postpartum hemorrhage, (5) hematological and neoplastic disorders, (6) severe liver diseases, (7) incomplete medical records $\geq 30 \%$, and (8) death before childbirth. According to these criteria, only 1587 of these deliveries were available and eligible for study. The final dataset included the 49 potential predictor variables and bleeding or not outcomes of these cases. Next, these women were randomly divided into a training set (70\%), a validation set (15\%) and a test set (15\%).

\subsection{Pre-processing and feature selection}

The outcomes event of postpartum hemorrhage was defined as blood loss at least $500 \mathrm{~mL}$ within 24 hours after the fetus is vaginally delivered. This outcome was selected because it was consistent with the most recent definition from "Guidelines for the Prevention and Management of Postpartum Hemorrhage 
(2014)" issued by Obstetrics Division, Obstetrics and Gynecology Branch, Chinese Medical Association [22], the literature of "obstetrics and gynecology" (published by People's Medical Publishing House in 2013, 8th Edition) [23], as well as the consensus definition of the Royal College of Obstetricians and Gynecologists ${ }^{[24]}$, and the World Health Organization [25].

There were data values missing for some variables, we used the multiple imputation to impute missing data values for variables in model. This approach has been recommended by the prediction model risk of bias assessment tool (PROBAST) ${ }^{[26]}$ and applied in previous study ${ }^{[27]}$.

Numerous variables will lead to over-fitting of the model and increase the clinic burden. Therefore, the preprocessing techniques were frequently used to reduce dimension or select feature. This step can eliminate irrelevant, weakly relevant, or less important features. Variable screening is an important procedure to improve the overall stability of the model. We selected variables using the "backward method" and the univariate logistic regression performed by IBM SPSS software (version 20). 7 variables were finally identified as input neurons, and the standardized importance of each variable was shown on a scale with a maximum value of 100 , to understand the contribution of each variable in the machine learning model (Fig. 1).

\subsection{Establishment of artificial neural network models and logistic regression (LR) model}

The artificial neural networks and LR models of postpartum hemorrhage were built based on the identified predictors from the previous step.

\subsubsection{The multi-layer perceptron (MLP) network}

The MLP neural network consists of one input layer, one or more hidden layer and one output layer. The neuron is the basic component of the network. Neurons include input neurons, hidden layer neurons and output neurons. MLP is a multi-layer feedforward neural network, it has advantages of processing nonlinear data, good fault tolerance and strong self-learning ability ${ }^{[17]}$. The MLP network model can pass the data among each neuron in direction, and excavate the internal deep connections between the input data, whose learning process mainly includes the forward transmission of data and the back propagation of errors. This kind of feedback learning method can reasonably change the weight and bias of each input so that obtain the best prediction ability for the model ${ }^{[28]}$. The number of neurons in hidden layer can be automatically calculated by SPSS software and the relative optimal number of neurons can be get.

\subsubsection{The Back Propagation (BP) network}

BP neural network is multi-layer feedforward neural network based on gradient descent algorithm, it is trained according to the error back propagation algorithm, and has been the most widely used neural network, which is composed of input layer, hidden layer and output layer. Activation function between 
layer and layer is Sigmoid type differentiable function, the error is propagated back to the input layer from back to front layer by layer, the link weight is adjusted constantly in order to realize arbitrary nonlinear mapping between input and output ${ }^{[19,29,30]}$. The internal Sigmoid function was automatically calculated by SPSS and the relative optimal function parameters were obtained.

\subsubsection{The radial basis function (RBF) network}

The RBF neural network consists of input layer, radial basis layer and output layer, the input layer is made up of signal source nodes; the radial basis layer is a nonlinear radially symmetric function that is attenuated to the center point according to specific needs; the output layer is the output result of input layer processed by radial basis function ${ }^{[21]}$. RBF neural network is a kind of forward conduction neural network which is applicable to solve classification problems. It can approximate any continuous function with any precision so that achieve the optimal prediction ability by adjusting the parameters of the radial basis function ${ }^{[30]}$. The internal radial basis function was automatically calculated by SPSS and the relative optimal function parameters were obtained.

\subsection{Evaluation of the networks and development of the CDSS}

Firstly, in order to identify the best-performed model based on a given dataset, we not only developed five models for the MLP network, RBF network and BP network, respectively, but also constructed the binary logistic regression to compare the performance of the traditional statistical analysis model and the artificial neural network model. Secondly, the receiver operating characteristic (ROC) curves of each model in training set, validation set, test set were plotted by R 3.6.3 software. We used the area under the receiver operating characteristic (AUROC), sensitivity, specificity, and accuracy to evaluate and select the most accurate model. In addition, we also conducted the internal validation by 10 -fold cross. Finally, we designed the CDSS based on the most accurate network by MATLAB 2013b software to reach visualization.

\subsection{Ethical and legal considerations}

This study was approved by the Ethics Committee of the Third Affiliated Hospital of Zhengzhou University and followed the ethical guidelines set by the Helsinki Declaration of the World Medical Congress (2019-135-01).

\section{Results}

\subsection{Identifying significant variables for predicting postpartum hemorrhage in vaginal delivery}

Originally, we identified 54 variables that is likely to affect the postpartum hemorrhage among vaginal delivery women (Appendix 1). Among the 1587 deliveries included, 1307 (82.4\%) just occurred 
physiological blood loss (less than $500 \mathrm{ml}$ ), in addition, there were $280(17.6 \%)$ cases of postpartum hemorrhage (31 of blood loss more than $1000 \mathrm{ml}$ and 249 of blood loss between $500-1000 \mathrm{ml}$ ).

After performing the univariable logistic regression, the most significant fifteen factors with $\mathrm{P}$-value $<0.01$ were selected for predicting the postpartum hemorrhage (Table 1). Tables 1 demonstrated the distribution of these factors in our total dataset for bleeding and not bleeding, respectively. Figure 1 showed the variable importance in the selected MLP model which had the best discrimination ability. The top 10 variables, ranked from most to least important, were white blood cells (WBC) during the first labor stage, newborn weight, cervical laceration, history of uterine surgery, parity, manual removal of placenta, episiotomy, placenta previa, operative vaginal delivery, uterine curettage, assisted reproduction, velamentous placenta, threatened abortion, induced labor, and placental abruption.

Table 1

Descriptive statistics of variables

\begin{tabular}{|llll|}
\hline Variables & \multicolumn{2}{l}{ Postpartum Hemorrhage } & \multirow{2}{*}{ P-value } \\
\cline { 2 - 3 } & No $(\mathbf{n = 1 3 0 7 )}$ & Yes $(\mathbf{n = 2 8 0})$ & \\
\hline Threatened Abortion & $73(5.59)$ & $53(18.89)$ & $<0.01$ \\
\hline History of Uterine Surgery & $0.08 \pm 0.29$ & $0.22 \pm 0.56$ & $<0.01$ \\
\hline Parity & $0.54 \pm 0.63$ & $0.44 \pm 0.60$ & $<0.01$ \\
\hline Placenta Previa & $5(0.38)$ & $6(2.14)$ & $<0.01$ \\
\hline Velamentous Placenta & $42(3.21)$ & $24(8.57)$ & $<0.01$ \\
\hline Induced Labor & $102(7.80)$ & $36(12.68)$ & $<0.01$ \\
\hline Assisted Reproduction & $30(2.30)$ & $17(6.07)$ & $<0.01$ \\
\hline Newborn Weight & $3252.95 \pm 521.42$ & $3385.21 \pm 613.22$ & $<0.01$ \\
\hline Operative Vaginal Delivery & $18(1.38)$ & $22(7.86)$ & $<0.01$ \\
\hline Manual Removal of Placenta & $61(4.67)$ & $40(14.29)$ & $<0.01$ \\
\hline Uterine Curettage & $918(70.24)$ & $242(86.43)$ & $<0.01$ \\
\hline Episiotomy & $297(22.72)$ & $117(41.79)$ & $<0.01$ \\
\hline Cervical Laceration & $44(3.37)$ & $34(12.14)$ & $<0.01$ \\
\hline Placental Abruption & $8(0.61)$ & $9(3.21)$ & $<0.01$ \\
\hline WBC during the first stage of labor & $10.33 \pm 3.48$ & $15.13 \pm 4.53$ & $<0.01$ \\
\hline
\end{tabular}




\subsection{Designing and evaluating BP, MLP and RBF neural networks}

Table 2

Results of evaluating MLP, BP and RBF networks with different neurons (based on total data)

\begin{tabular}{|c|c|c|c|c|c|c|}
\hline ANN & $\begin{array}{l}\text { Number of } \\
\text { neurons } \\
\text { (hidden layer) }\end{array}$ & $\begin{array}{l}\text { Functions } \\
\text { (hidden layer) }\end{array}$ & $\begin{array}{l}\text { Functions } \\
\text { (output } \\
\text { layer) }\end{array}$ & $\begin{array}{l}\text { Accuracy } \\
(\%)\end{array}$ & $\begin{array}{l}\text { Specificity } \\
(\%)\end{array}$ & $\begin{array}{l}\text { Sensitivity } \\
(\%)\end{array}$ \\
\hline \multirow[t]{5}{*}{ MLP } & 6 & \multirow{5}{*}{$\begin{array}{l}\text { Hyperbolic } \\
\text { Tangent }\end{array}$} & \multirow[t]{5}{*}{ Softmax } & 91.5 & 95.2 & 73.6 \\
\hline & 8 & & & 92.3 & 94.8 & 80.4 \\
\hline & 6 & & & 93.3 & 95.7 & 83.2 \\
\hline & 8 & & & 93.8 & 95.4 & 86.9 \\
\hline & 8 & & & 94.3 & 94.9 & 90.0 \\
\hline \multirow[t]{5}{*}{ BP } & 10 & \multirow[t]{5}{*}{ Sigmoid } & \multirow[t]{5}{*}{ Sigmoid } & 86.4 & 96.0 & 41.4 \\
\hline & 10 & & & 92.1 & 94.9 & 79.3 \\
\hline & 8 & & & 86.0 & 95.7 & 59.6 \\
\hline & 10 & & & 89.0 & 95.9 & 71.8 \\
\hline & 8 & & & 86.9 & 95.1 & 51.4 \\
\hline \multirow[t]{5}{*}{$\mathrm{RBF}$} & 6 & \multirow[t]{5}{*}{ Softmax } & \multirow[t]{5}{*}{ Identity } & 84.0 & 95.5 & 30.2 \\
\hline & 9 & & & 84.8 & 95.0 & 36.8 \\
\hline & 5 & & & 87.5 & 94.9 & 46.2 \\
\hline & 5 & & & 84.7 & 94.2 & 40.1 \\
\hline & 10 & & & 83.7 & 95.3 & 29.6 \\
\hline
\end{tabular}

\subsubsection{MLP structure}

The multi-layer perceptron (MLP) network consisted of Hyperbolic Tangent activation functions in the hidden layer, Softmax activation functions in the output layer. Table 2 demonstrated the number of neurons in the hidden layer and performance of the five trained networks. As seen, Network 5 performed the best, the accuracy, specificity, and sensitivity (in the total data) was $94.3 \%, 94.9 \%$, and $90.0 \%$, respectively, there were 8 epochs in the hidden layer. 
As shown in confusion matrix of Table 3 , the computer randomly selected $70 \%$ of the total sample size (1111 cases) for the model training, 15\% (234 cases) for the model validation, and the rest $15 \%$ (241 cases) for the model test. For the 1111 samples used for training, there were 912 samples with nonhemorrhage, 858 cases were accurately predicted with specificity of $94.1 \%$. The outcome of the remaining 199 samples was postpartum hemorrhage, of which 180 cases were predicted accurately, with sensitivity of $90.5 \%$. The overall prediction accuracy of the training was $93.4 \%$. Similarly, in the validation set, the specificity, sensitivity, accuracy was $94.8 \%, 90.0 \%, 94.0 \%$, respectively; in the test set, that was $94.0 \%, 87.8 \%, 92.9 \%$, respectively.

Table 3

Confusion matrix of the MLP

\begin{tabular}{|c|c|c|c|c|c|}
\hline & & Non-bleeding & Bleeding & & \\
\hline \multirow[t]{3}{*}{ Training set (70\%) } & Non-bleeding & 858 & 54 & $94.1 \%$ & Specificity \\
\hline & Bleeding & 19 & 180 & $90.5 \%$ & Sensitivity \\
\hline & Total (\%) & 78.9 & 21.1 & $93.4 \%$ & Accuracy \\
\hline \multirow[t]{3}{*}{ Validation set (15\%) } & Non-bleeding & 184 & 10 & $94.8 \%$ & Specificity \\
\hline & Bleeding & 4 & 36 & $90.0 \%$ & Sensitivity \\
\hline & Total (\%) & 80.3 & 19.7 & $94.0 \%$ & Accuracy \\
\hline \multirow[t]{3}{*}{ Test set (15\%) } & Non-bleeding & 188 & 12 & $94.0 \%$ & Specificity \\
\hline & Bleeding & 5 & 36 & $87.8 \%$ & Sensitivity \\
\hline & Total (\%) & 80.1 & 19.9 & $92.9 \%$ & Accuracy \\
\hline
\end{tabular}

\subsubsection{BP structure}

The Back Propagation (BP) network covered Sigmoid activation functions in both hidden layer and output layer. Table 2 revealed the number of neurons in the hidden layer and performance of the five trained networks. As seen, Network 7 performed the best, accuracy, specificity and sensitivity (in the total data) was $92.1 \%, 94.9 \%$, and $79.3 \%$, respectively, there were 10 epochs in the hidden layer.

As shown in confusion matrix of Table 4 , the computer randomly selected $70 \%$ of the total sample size ( 1116 cases) for the model training, $15 \%$ (240 cases) for the model validation, and the rest $15 \%$ ( 231 cases) for the model test. For the 1116 samples used for training, there were 926 samples with nonhemorrhage, 877 cases were accurately predicted with specificity of $94.7 \%$. The outcome of the remaining 190 samples was postpartum hemorrhage, of which 150 cases were predicted accurately with sensitivity of $78.9 \%$. The overall prediction accuracy of the training was $92.0 \%$. Similarly, in the validation 
set, the specificity, sensitivity, accuracy was $96.3 \%, 79.6 \%, 92.9 \%$, respectively; in the test set, that was $94.2 \%, 81.0 \%, 91.8 \%$, respectively.

Table 4

Confusion matrix of the BP

\begin{tabular}{|llllll|}
\hline \multicolumn{5}{c|}{ Non-bleeding } & Bleeding \\
\hline Training set (70\%) & Non-bleeding & 877 & 49 & $94.7 \%$ & Specificity \\
\cline { 2 - 6 } & Bleeding & 40 & 150 & $78.9 \%$ & Sensitivity \\
\cline { 2 - 6 } & Total (\%) & 82.2 & 17.8 & $92.0 \%$ & Accuracy \\
\hline Validation set (15\%) & Non-bleeding & 184 & 7 & $96.3 \%$ & Specificity \\
\cline { 2 - 6 } & Bleeding & 10 & 39 & $79.6 \%$ & Sensitivity \\
\cline { 2 - 6 } & Total (\%) & 80.8 & 19.2 & $92.9 \%$ & Accuracy \\
\hline Test set (15\%) & Non-bleeding & 178 & 11 & $94.2 \%$ & Specificity \\
\cline { 2 - 6 } & Bleeding & 8 & 34 & $81.0 \%$ & Sensitivity \\
\cline { 2 - 6 } & Total (\%) & 80.5 & 19.5 & $91.8 \%$ & Accuracy \\
\hline
\end{tabular}

\subsubsection{RBF structure}

The radial basis function (RBF) network was composed of Softmax activation functions in the hidden layer, Identity activation functions in the output layer. Table 2 indicated the number of neurons in the hidden layer and performance of the five trained networks. As seen, Network 13 performed the best, accuracy, specificity, and sensitivity (in the total data) was $87.5 \%, 94.9 \%$, and $46.2 \%$, respectively, there were 5 epochs in the hidden layer.

According to Table 5, the computer randomly selected $70 \%$ of the total sample size (1129 cases) for the model training, 15\% (228 cases) for the model validation, and the rest 15\% (223 cases) for the model test. For the 1129 samples used for training, there were 936 samples with non-hemorrhage, 898 cases were accurately predicted with specificity of $95.9 \%$. The outcome of the remaining 193 samples was postpartum hemorrhage, of which 88 cases were predicted accurately with sensitivity of $45.6 \%$. The overall prediction accuracy of the training was $87.3 \%$. Similarly, in the validation set, the specificity, sensitivity, accuracy was $96.2 \%, 45.2 \%, 86.8 \%$, respectively; in the test set, the specificity, sensitivity, accuracy was $97.8 \%, 50.0 \%, 89.2 \%$, respectively. 
Table 5

Confusion matrix of the RBF

\begin{tabular}{|llllll|}
\hline \multicolumn{5}{|c|}{ Non-bleeding } & Bleeding \\
\hline Training set (70\%) & Non-bleeding & 898 & 38 & $95.9 \%$ & Specificity \\
\cline { 2 - 6 } & Bleeding & 105 & 88 & $45.6 \%$ & Sensitivity \\
\cline { 2 - 6 } & Total (\%) & 88.8 & 11.2 & $87.3 \%$ & Accuracy \\
Validation set (15\%) & Non-bleeding & 179 & 7 & $96.2 \%$ & Specificity \\
\cline { 2 - 6 } & Bleeding & 23 & 19 & $45.2 \%$ & Sensitivity \\
\cline { 2 - 6 } Test set (15\%) & Total (\%) & 88.6 & 11.4 & $86.8 \%$ & Accuracy \\
& Non-bleeding & 179 & 4 & $97.8 \%$ & Specificity \\
\cline { 2 - 6 } & Bleeding & 20 & 20 & $50.0 \%$ & Sensitivity \\
\cline { 2 - 6 } & Total (\%) & 89.2 & 10.8 & $89.2 \%$ & Accuracy \\
\cline { 2 - 6 }
\end{tabular}

\subsubsection{Developing the CDSS}

To compare the performance of MLP, BP, RBF, and LR model, we put the four ROC curves of the four models together in the training set, validation set, and test set, respectively (Fig. 2). As shown in the Table 6 and Fig. 2, after the validation, best performing model was the multi-layer perceptron (MLP) network with the highest discriminative ability (AUC $0.862,95 \% \mathrm{Cl} 0.838-0.887$ ). Followed by the BP model (AUC $0.866 ; 95 \% \mathrm{Cl} 0.842-0.890$ ), logistic regression (AUC 0.856; 95\% Cl 0.832-0.880). The RBF model (AUC $0.845 ; 95 \% \mathrm{Cl} 0.820-0.870$ ) had lower discriminative ability. The MLP network had the best performance in the training data and test data, as well (Table 6).

Table 6

AUC of the MLP, BP, RBF network and LR in the training/validation/test data

\begin{tabular}{|llll|}
\hline & Training set & Validation set & Test set \\
\hline MLP & $0.871(0.847-0.894)$ & $0.862(0.838-0.887)$ & $0.870(0.847-0.894)$ \\
\hline BP & $0.866(0.842-0.890)$ & $0.866(0.842-0.890)$ & $0.865(0.840-0.889)$ \\
RBF & $0.847(0.823-0.872)$ & $0.845(0.820-0.870)$ & $0.826(0.798-0.853)$ \\
\hline LR & $0.851(0.825-0.877)$ & $0.856(0.832-0.880)$ & $0.853(0.828-0.877)$ \\
\hline
\end{tabular}

Finally, we selected the Network 5 of MLP for developing the CDSS. The graphical user interface of this CDSS was displayed in Fig. 3. As seen in this figure, obstetricians can easily input the values of the 15 variables and get the result of prediction for the postpartum hemorrhage 


\section{Discussion}

We preliminarily constructed and validated three ANN models to predict postpartum hemorrhage in vaginal delivery women. The MLP model indicated the best predictive ability in all groups of training, validation and test. Moreover, compared with the traditional logistic regression model, both MLP and BP models showed better discrimination ability to predict postpartum hemorrhage. In addition, A userfriendly CDSS was developed according to the MLP model.

In terms of clinical application, midwives could prepare full in advance for example triage expectant mothers with high risk of postpartum hemorrhage to the corresponding obstetric care level and initiate interventions. Prediction of our model mainly focused on the time of labor and birth, therefore, we adopted the variables available during the same instances. It is also appropriate to construct prediction model employing high-risk factors after delivery, such as cervical lacerations and newborn weight. Importantly, in order to facilitate clinical use immediately, we recommend the information departments in hospital to integrate the aid decision-making system in electronic medical records, in the form of a risk calculator or outputting the result automatically. When a pregnant woman is laboring by an obstetrician, the midwife or other assistant could input the numerical values of the 15 predictors into the built CDSS (Fig. 3), and the prediction outcome (postpartum hemorrhage or non-postpartum hemorrhage) would be output by clicking the button of "submit" in the designed interface of the system. Once the system identifies some pregnancy women as postpartum hemorrhage, the one should be considered carefully by the obstetricians. According to the predicted outcome, the obstetricians can make optimal decision for the pregnancy women about personalized treatment in the next step. In this way, the obstetrician can manage the labor stage more skillfully by early identification, early warning, early treatment, in order to mitigate adverse outcome for these high-risk patients. For example, midwives can make pre-transfusion preparations for high-risk patients in advance, cross-match blood tests, and inform the blood transfusion department to prepare blood products, particularly fresh frozen plasma can take up an hour to thaw. At the same time, it is conducive to the rational allocation of medical resources by arranging high-risk bleeding women for delivering in different time to avoid insufficient staff resources.

In recent years, medical institutions and obstetric experts at home and abroad have made a lot of exploration in the early warning evaluation and prediction of postpartum hemorrhage. The risk score calculators formulated by them for different subgroups of people could indicate the possibility of postpartum hemorrhage to some extent and achieved certain results. Ana ${ }^{[31]}$ developed and validated a predictive model based on the binary logistic regression and the ridge regression to measure the risk of excessive blood loss in 2336 vaginal delivery women, but this analysis only collected thirteen variables and the sensitivity was low. Michelle ${ }^{[32]}$ trained and tested a logistic regression prediction models involving 74 variables for hemorrhage and transfusion by a data set from 63973 deliveries, however, the results were not visualized. Kartic ${ }^{[15]}$ et al. used two traditional statistical models (logistic regression with and without lasso regularization) and two machine learning models (random forest and extreme gradient boosting) to predict postpartum hemorrhage, the extreme gradient boosting model showed the best in both the discrimination and decision curve analysis, but some variables in the model were difficult to 
collect so that limited the clinical practicability. Ahmadzia et al. ${ }^{[33]}$ developed an online calculator for postpartum hemorrhage risk scores, Dunkerton ${ }^{[34]}$ et al. established a decision tree model for postpartum hemorrhage prediction based on a non-parametric recursion algorithm, Bingnan Chen ${ }^{[35]}$ created a nomogram model to predict postpartum hemorrhage individually, however, all of them were only used for cesarean section population. Due to the heterogeneity of discriminant criteria and risk factors between cesarean section and vaginal delivery, there are some limitations in predicting postpartum hemorrhage using the same warning model for different mode of delivery. In addition, most of current prediction models for postpartum hemorrhage were linear algorithms, logistic regression is a traditional statistics algorithm which can screen out the limited variables associated with postpartum hemorrhage and eliminate confounding variables ${ }^{[36]}$. However, when there are too many variables to observe, the LR neither detect complicated nonlinear relationships between independent and dependent variables, nor has the ability to address collinearity between variables, so some potential valid variables were removed ${ }^{\text {[37] }}$. Compare with above models, our study indicated that both neural network and logistic regression can provide excellent discrimination for the prediction of postpartum hemorrhage, hence, a finally selected model to build a CDSS should rely on a combination of model performance (discrimination, calibration and net benefit), clinical applicability, and acceptability by obstetricians and expectant women ${ }^{[38]}$.

The ANN prediction model established in this paper aims to provide personalized prediction results and achieve effective risk stratification. The results of our study revealed that the ANN model was more accurate to predict postpartum hemorrhage among vaginal delivery. At present, the ANN model has been widely used in disease prediction and diagnosis, chronic disease management, medical image recognition and other aspects ${ }^{[16-21]}$, it had shown superior performance than conventional predictive models even employing the same input variables ${ }^{[16,21]}$. Postpartum hemorrhage, as an obstetric emergency, has various factors and complex mechanism. If we take the epidemiological data and use the traditional linear discriminant function to predict it, there are great limitations. As an information processing system abstracted from biological neural network, ANN has the ability of self-learning and identifying the relationship between variables, which can approximate arbitrary nonlinear functions with arbitrary accuracy ${ }^{[17]}$. Previous studies implied that neural network models reflected a stronger fit to address complex nonlinear relationships and cost less effort to generate than the traditional regression algorithms ${ }^{[18-21]}$. It is imperative that ANN techniques automatically conduct variables selection, missing values imputing and other data preprocessing procedures.

There was a lot of literature support for the included variables as risk factors for PPH. Almost all the variables in our model are clinically available and have been identified in previous models, including newborn weight, cervical laceration, history of uterine surgery, parity, manual removal of placenta, episiotomy ${ }^{[31-35,38]}$. However, one important predictor that has not been considered yet is the WBC count during the first stage of labor, it is identified of $100 \%$ importance in this study, which extended the previous work in predicting PPH and may provide insights of underlying pathophysiology links between inflammation participation and PPH onset. In our results, the WBC count of hemorrhage women were higher than that of non-hemorrhage women during the first stage of labor. Possible reasons for this may 
be as follows: infection leads to weak state of pregnancy women and those were vulnerable to $\mathrm{PPH}$, and labor is a state of stress which also leads high WBC count ${ }^{[39,40]}$. Further, more clinical laboratory studies are needed to uncover the underlying pathophysiology mechanism between high WBC count during the first stage of labor and postpartum hemorrhage. Our outcomes for the WBC showed a 100\% standardized importance, this point maybe because the sample size was large, but the morbidity of PPH was low and the number of cases was small in this model. Nevertheless, another statistical algorithm was conducted (binary logistic regression) to explore the odds ratio (OR) of the variables and confirm their statistical significance.

The second important risk factor was the newborn weight of $75.5 \%$ standardized importance in our study, which again matched previous studies $[13,15,24,31]$. This may because that increased newborn weight is associated with hyperextension of uterine muscle fibers and affects uterine contractions. In addition, pregnant women with large fetal weight may have other complications, such as cephalic pelvic asymmetricity, prolonged first and second stages of labor, shoulder dystocia, laceration of the soft birth canal, uterine contraction ${ }^{[41]}$, which also increases the incidence of postpartum hemorrhage. The newborn weight in our study was postpartum variable when the risk period of bleeding had passed, we can consider introducing a prenatal diagnosis of macrosomia or estimated fetal weight according to the ultrasound into model. Some other risk factors in our model for hemorrhage have been accepted generally, namely operative vaginal delivery, induced labor, manual removal of placenta, episiotomy and cervical tears $[2,3,6,31-35]$, despite their fewer links to hemorrhage with standardized importance less than $30 \%$, the less noticeable whose effect is, the more severe the situation goes.

\subsection{Limitations}

Our study has several limitations. Firstly, neural network algorithms are driven by big data and rely on a large sample size. We developed a CDSS based on electronic medical record from only one center, the sample size was far insufficient, the external validation was just conducted in our hospital, so the result may be not generalizable, robust internal and external validation is needed before promotion and application widely. Secondly, Missing data is another limitation of our study, the study was restricted in the part of valid blood loss data from the original dataset, the missing value of the covariable accounted for a considered proportion, though we adopted multiple imputation techniques reported by the PROBAST [26], the proportion of incomplete data also limited the generalization of the model. Furthermore, it is more likely that the missing values will continue to hinder integrating the models into electronic medical records. Thirdly, the predictors in our study all were static, hourly data such as temperature, heart rate, systolic blood pressure, oxygen saturation, physical examination findings were not included.

In addition, despite embedding all risk factors in the CDSS will be promising, but it may increase clinical workload burden and could potentially delay intervention ${ }^{[42]}$. The impact of a model on aiding decision making depends on multiple characteristics of health providers and circumstances, including ability to initiate immediately intervene response and weigh the risks against benefits, capacity to take actions, and 
expectant women (or obstetricians) compliance with the recommended measures ${ }^{[43]}$. Other environmental restrictions consist of staff, space, and facilities, which are not considered into current CDSS. Meanwhile, estimated blood loss (EBL) is considered to be inaccurate, subjective and always underestimated, there is a mismatch between the actual blood loss and the vital signs, urine volume and mental state of the individual shown. A physical compensatory period of blood loss exists in the early bleeding stage ${ }^{[44-45]}$. Our definition of postpartum hemorrhage followed the current clinical guidelines ${ }^{[22-25]}$, however, we did not evaluate other relevant clinical indicators of acute blood loss, including a deep perineal hematoma from a laceration of the birth canal, blood flow velocity and properties, the bleeding was fluid or turbulent or exudative, and whether it clotted or contained clots ${ }^{[46]}$. The blood loss in predictive models needs to be measured by means of the quantitative blood loss (QBL) methods, such as basic methemoglobin colorimetric method ${ }^{[47]}$ or image spectral analysis ${ }^{[45]}$, Shock Index (SI) ${ }^{[48]}$ and so on.

\section{Conclusions}

In summary, we developed a CDSS based on the risk factors identified by artificial neural network algorithms. This ANN model performed better than logistic regression in predicting the postpartum hemorrhage of vaginal delivery women. In the future, these findings will enrich nursing informational practice to promote the application of artificial intelligence technology (such as ANN algorithm) in the field of nursing. As predictive tools become more widely used in obstetric care, they can be introduced into clinical guidelines and care pathways after further testing. Identification high risk mother of postpartum hemorrhage on labor admission using the clinical decision-making system will benefit accurate prenatal diagnosis and prompt intervention, which may lead to optimize obstetric care, improve maternal prognosis, and rational allocation of medical resource.

\section{Abbreviations}

CDSS

Clinical decision support system; MLP:Multi-layer perceptron; BP:Back propagation; RBF:Radial basis function; LR:Logistic regression; ROC:Receiver operating characteristic; ANN:Artificial neural network; PPH:Postpartum hemorrhage; TOLAC:Trial of labor after a previous cesarean section; ERCS:Elective repeated cesarean section; ACOG:the American College of Obstetricians and Gynecologists; CMQCC:the California Maternal Quality Care Collaborative; AUROC:Area under the receiver operating characteristic; WBC:White blood cells; OR:Odds ratio; EBL:Estimated blood loss; QBL:Quantitative blood loss.

\section{Declarations}

\section{Ethics approval and consent to participate}

This study was approved by the Ethics Committee of the Third Affiliated Hospital of Zhengzhou University and followed the ethical guidelines set by the Helsinki Declaration of the World Medical 
Congress (2019-135-01). Informed consent was obtained by telephone from all the subjects before data collection and the private information in the study was well protected.

\section{Consent for publication}

Not applicable.

\section{Availability of data and materials}

The datasets used and/or analyzed during the current study are available from the corresponding author on reasonable request.

\section{Competing interest}

The authors declare no competing interest.

\section{Funding}

This research is supported by the joint construction project of Henan Province medical science and technology research plan (No. SBGJ2018049).

\section{Authors' contributions}

YJ, GP, SY, HL, YX and BH made substantial contributions to conception and design, or acquisition of data, or analysis and interpretation of data. YJ, SY, HL and YX involved in drafting the manuscript or revising it critically for important intellectual content. GP completed the data analysis. BH served as a consultant to others and directed them to complete the experimental design. Each author should have participated sufficiently in the work to take public responsibility for appropriate portions of the content. YJ and $\mathrm{BH}$ agreed to be accountable for all aspects of the work in ensuring that questions related to the accuracy or integrity of any part of the work are appropriately investigated and resolved.

\section{Acknowledgements}

We would like to thank all participants for their contribution to the study.

\section{ORCID}

Jingxuan Yang https://orcid.org/0000-0002-7102-1285

Hua Bai https://orcid.org/0000-0003-4944-7156

\section{References}

1. National Health and Family Planning Commission. China Health and Family Planning Statistical Yearbook 2019. Beijing: Peking Union Medical College Press. 2019. 
2. Sentilhes L, Merlot B, Madar H, Sztark F, Brun S, Deneux-Tharaux C. Postpartum haemorrhage: prevention and treatment. Expert Rev Hematol. 2016;9(11):1043-1061. doi: 10.1080/1747 4086.2016.12 45135.

3. Borovac-Pinheiro A, Pacagnella RC, Cecatti JG, Miller S, El Ayadi AM, Souza JP, Durocher J, Blumenthal PD, Winikoff B. Postpartum hemorrhage: new insights for definition and diagnosis. Am J Obstet Gynecol. 2018;219(2):162-168. doi: 10.1016/j.ajog.2018.04.013.

4. Appropriate technology for birth. Lancet. 1985;2(8452):436-7.

5. Yang J, Bai H. Knowledge, attitude and experience of episiotomy practice among obstetricians and midwives: a cross-sectional study from China. BMJ Open. 2021;11(4):e043596. doi:

10.1136/bmjopen-2020-043596.

6. Practice Bulletin No. 183 Summary: Postpartum Hemorrhage. Obstet Gynecol. 2017;130(4):923-925. doi: 10.1097/AOG.0000000000002346.

7. Bright TJ, Wong A, Dhurjati R, Bristow E, Bastian L, Coeytaux RR, Samsa G, Hasselblad V, Williams JW, Musty MD, Wing L, Kendrick AS, Sanders GD, Lobach D. Effect of clinical decision-support systems: a systematic review. Ann Intern Med. 2012;157(1):29-43. doi: 10.7326/0003-4819-157-1201207030-00450.

8. Shortliffe EH, Sepúlveda MJ. Clinical Decision Support in the Era of Artificial Intelligence. JAMA. 2018;320(21):2199-2200. doi: 10.1001/jama.2018.17163.

9. Montani S, Striani M. Artificial Intelligence in Clinical Decision Support: a Focused Literature Survey. Yearb Med Inform. 2019;28(1):120-127. doi: 10.1055/s-0039-1677911.

10. State Council of the People's Republic of China. Opinions of the General Office of the State Council on Promoting the Development of "Internet + Medical Health". Guo Ban Fa [2018] No. 26. http://www.gov.cn/zhengce/content/2018-04/28/content_5286645.htm

11. National Health Commission of the People's Republic of China. Notice on Further Promoting the Informatization Construction of Medical Institutions Centered on Electronic Medical Records. The National Health Office [2018] No. 20.

http://www.nhc.gov.cn/yzygj/s7659/201808/a924c197326440cdaaa0e563f5b111c2.shtml

12. Garg AX, Adhikari NK, McDonald H, Rosas-Arellano MP, Devereaux PJ, Beyene J, Sam J, Haynes RB. Effects of computerized clinical decision support systems on practitioner performance and patient outcomes: a systematic review. JAMA. 2005;293(10):1223-38. doi: 10.1001/jama.293.10.1223.

13. Bateman BT, Berman MF, Riley LE, Leffert LR. The epidemiology of postpartum hemorrhage in a large, nationwide sample of deliveries. Anesth Analg. 2010;110(5):1368-73. doi: 10.1213/ANE.0b013e 3181d74898.

14. Main EK, Goffman D, Scavone BM, Low LK, Bingham D, Fontaine PL, Gorlin JB, Lagrew DC, Levy BS; National Partnership for Maternal Safety; Council on Patient Safety in Women's Health Care. National Partnership for Maternal Safety: Consensus Bundle on Obstetric Hemorrhage. Obstet Gynecol. 2015;126(1):155-62. doi: 10.1097/AOG.0000000000000869. 
15. Venkatesh KK, Strauss RA, Grotegut CA, Heine RP, Chescheir NC, Stringer JSA, Stamilio DM, Menard $\mathrm{KM}$, Jelovsek JE. Machine Learning and Statistical Models to Predict Postpartum Hemorrhage. Obstet Gynecol. 2020;135(4):935-944. doi: 10.1097/AOG.0000000000003759.

16. Garcia-Vidal C, Sanjuan G, Puerta-Alcalde P, Moreno-García E, Soriano A. Artificial intelligence to support clinical decision-making processes. EBioMedicine. 2019;46:27-29. doi: 10.1016/j.ebiom. 2019.07.019.

17. Kriegeskorte N, Golan T. Neural network models and deep learning. Curr Biol. 2019;29(7):R231-R236. doi: 10.1016/j.cub.2019.02.034.

18. Hassabis D, Kumaran D, Summerfield C, Botvinick M. Neuroscience-Inspired Artificial Intelligence. Neuron. 2017;95(2):245-258. doi: 10.1016/j.neuron.2017.06.011.

19. Kraft M, Gebauer J, Dölle-Bierke S, Worm M. Usefulness of an artificial neural network to assess anaphylaxis severity. Allergy. 2021;76(4):1244-1246. doi: 10.1111/all.14560.

20. Paydar K, Niakan Kalhori SR, Akbarian M, Sheikhtaheri A. A clinical decision support system for prediction of pregnancy outcome in pregnant women with systemic lupus erythematosus. Int $J$ Med Inform. 2017;97:239-246. doi: 10.1016/j.jimedinf.2016.10.018.

21. Mai RY, Lu HZ, Bai T, Liang R, Lin Y, Ma L, Xiang BD, Wu GB, Li LQ, Ye JZ. Artificial neural network model for preoperative prediction of severe liver failure after hemihepatectomy in patients with hepatocellular carcinoma. Surgery. 2020;168(4):643-652. doi: 10.1016/j.surg.2020.06.031.

22. Obstetrics Division, Obstetrics and Gynecology Branch, Chinese Medical Association. Guidelines for Prevention and Management of Postpartum Hemorrhage (2014). Chin J Obstet Gynecol. 2014,49 (9):641-646. doi: 10.3969/j.issn.1672-7185.2015.10.05

23. Xie Xing, Gou Wenli. Obstetrics and Gynecology (8th Edition). People's Medical Publishing House. 2013.

24. Royal College of Obstetricians and Gynaecologists. Postpartum haemorrhage, prevention and management (Green-top guideline No. 52). Available at: https://www.rcog.org.uk/en/guidelinesresearch-services/guidelines/gtg52/.

25. World Health Organization. The prevention and management of postpartum haemorrhage: report of a technical working group. Geneva, Switzerland: World Health Organization; 1989.

26. Wolff RF, Moons KGM, Riley RD, Whiting PF, Westwood M, Collins GS, Reitsma JB, Kleijnen J, Mallett S; PROBAST Groupt. PROBAST: A Tool to Assess the Risk of Bias and Applicability of Prediction Model Studies. Ann Intern Med. 2019;170(1):51-58. doi: 10.7326/M18-1376.

27. Sturdza AE, Poetter R, Kossmeier M, Kirchheiner K, Mahantshetty U, Haie-Meder C, Lindegaard JC, Jurgenliemk-Schulz I, Tan LT, Hoskin P, van Limbergen E, Gillham C, Segedin B, Tharavichitkul E, Iturre EV, Fokdal LU, Polterauer S, Kirisits C, Tanderup K. Nomogram predicting overall survival in locally advanced cervical cancer patients treated by radio-chemotherapy including image guided brachytherapy: a Retro-EMBRACE study. Int J Radiat Oncol Biol Phys. 2021,28: S0360-3016(21)0040 4 - 1. doi: 10.1016/j.ijrobp.2021.04.022. 
28. Javed F, Gilani SO, Latif S, Waris A, Jamil M, Waqas A. Predicting Risk of Antenatal Depression and Anxiety Using Multi-Layer Perceptrons and Support Vector Machines. J Pers Med. 2021;11(3):199. doi: 10.3390/jpm11030199.

29. Chen Y, Mao Y, Pan X, Jin W, Qiu T. Verification and comparison of three prediction models of ischemic stroke in young adults based on the back propagation neural networks. Medicine (Baltimore). 2021;100(11):e25081. doi: 10.1097/MD.0000000000025081.

30. Deng Y, Zhou X, Shen J, Xiao G, Hong H, Lin H, Wu F, Liao BQ. New methods based on back propagation (BP) and radial basis function (RBF) artificial neural networks (ANNs) for predicting the occurrence of haloketones in tap water. Sci Total Environ. 2021; 772:145534. doi: 10.1016/j.scitoten v.2021.145534.

31. Rubio-Álvarez A, Molina-Alarcón M, Arias-Arias Á, Hernández-Martínez A. Development and validation of a predictive model for excessive postpartum blood loss: A retrospective, cohort study. Int J Nurs Stud. 2018; 79:114-121. doi: 10.1016/j.ijnurstu.2017.11.009.

32. Pressly MA, Parker RS, Waters JH, Beck SL, Jeyabalan A, Clermont G. Improvements and limitations in developing multivariate models of hemorrhage and transfusion risk for the obstetric population. Transfusion. 2021;61(2):423-434. doi: 10.1111/trf.16216.

33. Ahmadzia HK, Phillips JM, James AH, Rice MM, Amdur RL. Predicting peripartum blood transfusion in women undergoing cesarean delivery: A risk prediction model. PLoS One. 2018;13(12): e0208417. doi: $10.1371 /$ journal.pone.0208417.

34. Dunkerton SE, Jeve YB, Walkinshaw N, Breslin E, Singhal T. Predicting Postpartum Hemorrhage (PPH) during Cesarean Delivery Using the Leicester PPH Predict Tool: A Retrospective Cohort Study. Am J Perinatol. 2018;35(2):163-169. doi: 10.1055/s-0037-1606332.

35. Chen B, Zhang L, Wang D, Li J, Hou Y, Yang T, Li N, Qiao C. Nomogram to predict postpartum hemorrhage in cesarean delivery for women with scarred uterus: A retrospective cohort study in China. J Obstet Gynaecol Res. 2020;46(9):1772-1782. doi: 10.1111/jog.14354.

36. Xu S, Guan LJ, Shi BQ, Tan YS, Zhang XJ. Recurrent Hemoptysis After Bronchial Artery Embolization: Prediction Using a Nomogram and Artificial Neural Network Model. AJR Am J Roentgenol. 2020;215(6):1490-1498. doi: 10.2214/AJR.20.22775.

37. Sperandei S. Understanding logistic regression analysis. Biochem Med (Zagreb). 2014;24(1):12-8. doi: 10.11613/BM.2014.003.

38. Neary C, Naheed S, McLernon DJ, Black M. Predicting risk of postpartum haemorrhage: a systematic review. BJOG. 2021;128(1):46-53. doi: 10.1111/1471-0528.16379.

39. Jonsdottir IH, Sjörs Dahlman A. MECHANISMS IN ENDOCRINOLOGY: Endocrine and immunological aspects of burnout: a narrative review. Eur J Endocrinol. 2019;180(3):R147-R158. doi: 10.1530/EJE18-0741.

40. Suresh PS, Koner BC. Effect of acute and chronic stress on leucocyte count: modulation by chlordiazepoxide. Immunopharmacol Immunotoxicol. 2012;34(4):586-9. doi: 10.3109/08923973.2011.639375. 
41. Fukami T, Koga H, Goto M, Ando M, Matsuoka S, Tohyama A, Yamamoto H, Nakamura S, Koyanagi T, To Y, Kondo H, Eguchi F, Tsujioka H. Incidence and risk factors for postpartum hemorrhage among transvaginal deliveries at a tertiary perinatal medical facility in Japan. PLoS One. 2019;14(1):e0208873. doi: 10.1371/journal.pone.0208873.

42. Escobar GJ, Soltesz L, Schuler A, Niki H, Malenica I, Lee C. Prediction of obstetrical and fetal complications using automated electronic health record data. Am J Obstet Gynecol. 2021;224(2):137-147.e7. doi: 10.1016/j.ajog.2020.10.030.

43. Escobar GJ, Gupta NR, Walsh EM, Soltesz L, Terry SM, Kipnis P. Automated early detection of obstetric complications: theoretic and methodologic considerations. Am J Obstet Gynecol. 2019;220 (4): 297-307. doi: 10.1016/j.ajog.2019.01.208.

44. Lertbunnaphong T, Lapthanapat N, Leetheeragul J, Hakularb P, Ownon A. Postpartum blood loss: visual estimation versus objective quantification with a novel birthing drape. Singapore Med J. 2016;57 (6):325-328. doi: 10.11622/smedj.2016107.

45. Merlin MA, Alter SM, Raffel B, Pryor PW 2nd. External blood loss estimation using the MAR Method. Am J Emerg Med. 2009;27(9):1085-90. doi: 10.1016/j.ajem.2008.07.039.

46. Buckland SS, Homer CS. Estimating blood loss after birth: using simulated clinical examples. Women Birth. 2007;20(2):85-8. doi: 10.1016/j.wombi.2007.01.001.

47. Doctorvaladan SV, Jelks AT, Hsieh EW, Thurer RL, Zakowski MI, Lagrew DC. Accuracy of Blood Loss Measurement during Cesarean Delivery. AJP Rep. 2017;7(2):e93-e100. doi: 10.1055/s-0037-1601382.

48. Sohn CH, Kim WY, Kim SR, Seo DW, Ryoo SM, Lee YS, Lee JH, Oh BJ, Won HS, Shim JY, Lim KS. An increase in initial shock index is associated with the requirement for massive transfusion in emergency department patients with primary postpartum hemorrhage. Shock. 2013;40(2):101-5. doi: 10.1097/SHK.0b013e31829b1778.

\section{Figures}




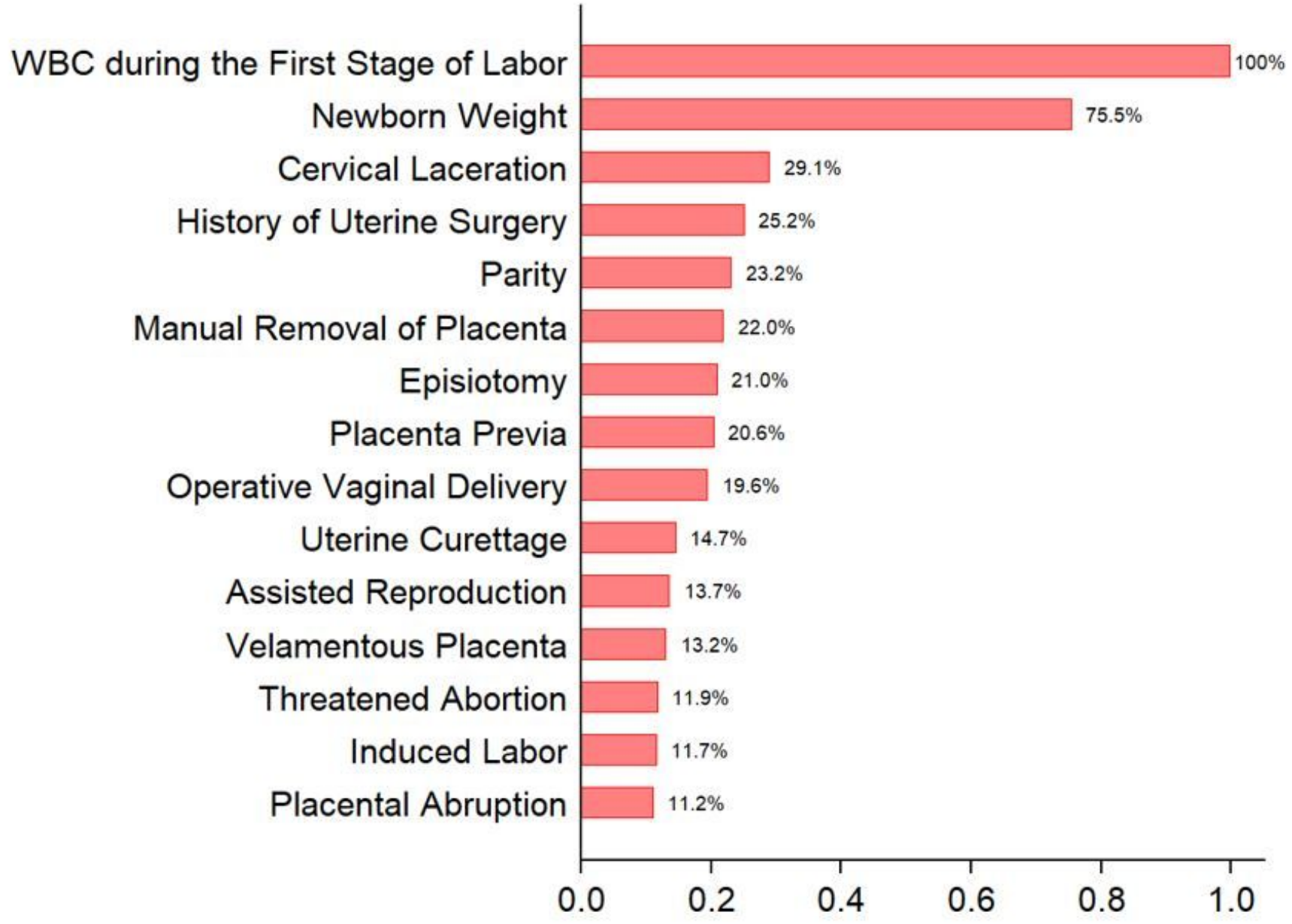

Figure 1

The standardized importance of the 15 factors in the MLP network 

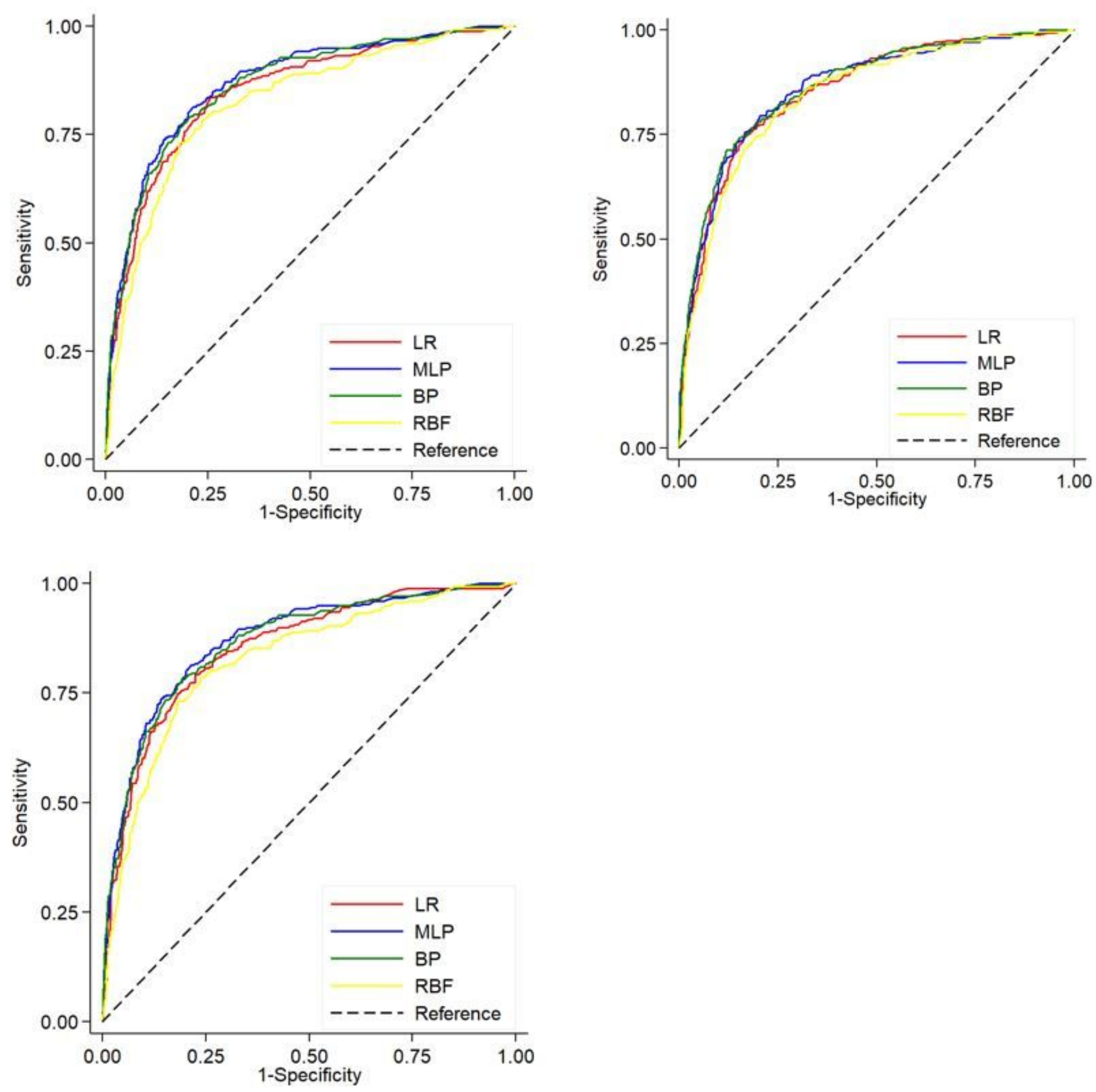

Figure 2

The ROC of the MLP, BP, RBF network and LR in the training/validation/test data 


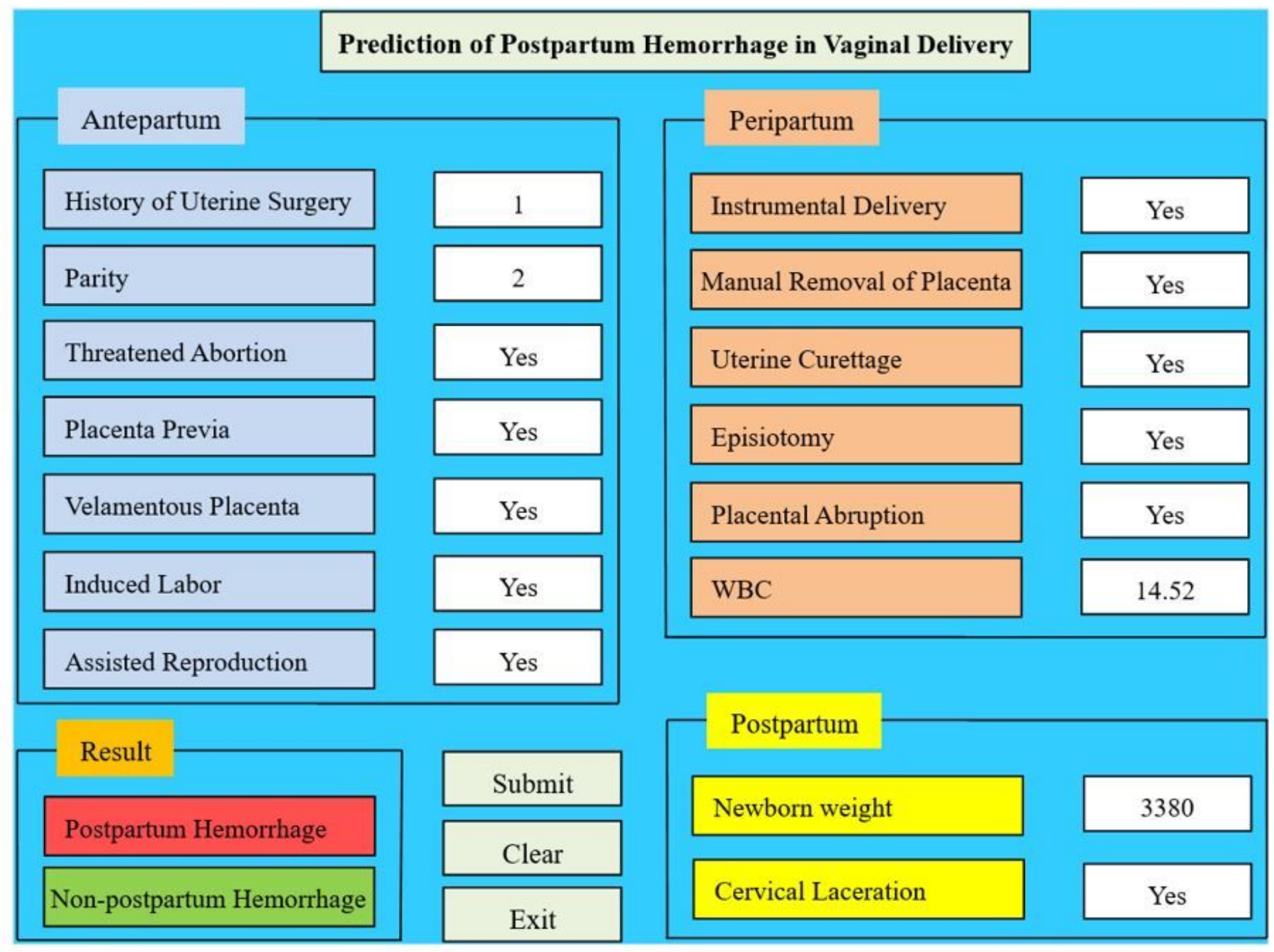

Figure 3

The graphical user interface of the CDSS developed for postpartum hemorrhage

\section{Supplementary Files}

This is a list of supplementary files associated with this preprint. Click to download.

- appendix.docx 